\title{
Effect of postnatal intermittent hypoxia on locomotor activity and neuronal development in rats tested in early adulthood
}

\author{
Karen Y. Yamada, Susan Y. Satake, Juliana C. Perry, Karina O. Garcia, Vânia D’Almeida, Sergio \\ Tufik, Beatriz M. Longo, and Regina C. Barbosa-Silva
}

Universidade Federal de São Paulo, São Paulo, SP, Brasil

\begin{abstract}
The present study evaluated the effects of postnatal intermittent hypoxia on locomotor activity and neuronal cell survival in early adulthood rats. During a critical period of brain development on postnatal day (PD) 7-11, male rat pups were exposed to intermittent hypoxia and randomly assigned to three experimental groups: (1) intermittent hypoxia, (2) normoxia, and (3) control (unhandled). One and a half months later on PD56, a behavioral test was conducted, and cell survival was estimated in the hilus, dental gyrus, and CA1 and CA3 subfields of the hippocampus, nucleus accumbens shell and core, dorsal and ventral striatum, and prefrontal cortex. Our results showed that intermittent hypoxia produced hyperactivity that correlated well with psychomotor agitation observed in patients with schizophrenia. Moreover, post-hypoxic rats exhibited a reduction of the number of neurons in the hilar region of the hippocampusanddorsalstriatum, structures thathavebeenneuropathologicallyassociatedwithschizophrenia. Thesefindingssuggest that intermittent hypoxia can modify the pattern of locomotor activity and selectively affect neurons in rats tested in early adulthood. Keywords: hypoxia, neurodevelopment, hyperactivity, cell survival, schizophrenia.
\end{abstract}

Received 06 November 2013; received in revised form 07 January 2014; accepted 07January 2014. Available online 15 May 2014.

\section{Introduction}

Schizophrenia is a complex neuropsychiatric disease that has genetic, environmental, and developmental aspects. The neurodevelopmental hypothesis of schizophrenia proposes that lesions caused by birth complications, infections, toxin exposure, or maternal stress in utero or during the postnatal period disturb the establishment of fundamental cerebral structural and functional aspects, rendering the brain vulnerable to adverse effects during puberty that precipitate the disease in young adulthood (Sandager-Nielsen, Andersen, Sager, Werge, \& Scheel-Kruger, 2004; Schwabe, Klein, \& Koch, 2006). A large number of epidemiological studies

Karen Y. Yamada, Susan Y. Satake, and Regina C. B. Silva, Laboratório de Psicologia Experimental, Departamento de Biociências, Universidade Federal de São Paulo. Juliana C. Perry, Vânia D'Almeida, and Sergio Tufik, Departamento de Psicobiologia, Universidade Federal de São Paulo. Karina O. Garcia, and Beatriz M. Longo, Laboratório de Neurofisiologia, Departamento de Fisiologia, Universidade Federal de São Paulo. Correspondence regarding this article should be directed to: Regina Cláudia Barbosa Silva, Departamento de Biociências, Universidade Federal de São Paulo, Av. D. Ana Costa, 95, Santos, São Paulo, CEP 11060-001, Brazil. Phone: +55 13 32218058. Fax: + 5513 32232592. E-mail: newstein@hotmail.com have demonstrated that perinatal complications, often involving fetal or neonatal hypoxia, are associated with an increased risk for the development of schizophrenia (McNeil, 1995; Cannon, 1997; Cantor-Graae, Ismail, \& McNeil, 2000; Vaillancourt, \& Boksa, 2000).

The slow process of rodent brain maturation enhances the vulnerability of neuronal survival. Some functional components do not fully mature until postnatal day (PD) 15-20 (Golan \& Huleihel, 2006). Exposure to intermittent hypoxia (IH) during this period was associated with marked increases in the number of apoptotic cells in the CA1 area of the hippocampus and cortex (Gozal, Daniel, \& Dohanich, 2001a; Gozal, Row, Schurr, \& Gozal, 2001b) and changes in the dopaminergic system (Decker, Hue, Caudle, Miller, Keating, \& Rye, 2003; Decker, Jones, Solomon, Keating, \& Rye, 2005). These alterations were related to cognitive deficits and increased motor activity (Gozal et al., 2001a; Row, Kheirandish, Neville, \& Gozal, 2002; Decker et al., 2005), both of which are considered clinical symptoms of schizophrenia.

Based on the assumption that the clinical features of schizophrenia typically appear in adolescence and early adulthood (Juarez, Silva-Gomez, Peralta, \& Flores, 2003), the aim of the present study was to investigate whether IH during a critical period of brain development (PD7-11) alters the pattern of locomotor activity and induces neuronal loss in early adulthood (PD56) in rats. 


\section{Methods}

\section{Subjects}

Male and female Wistar rats were mated in the animal facility of the Universidade Federal de São Paulo, São Paulo campus and allowed to deliver normally. Delivery times were recorded. The litters were culled to eight pups. PD1 was defined either as the day of delivery or as the next day if the pups were born after than 6:00 PM. Only males were used in this study, but female pups were allowed to stay in the litter until they were weaned. All of the rats were weaned at 4 weeks of age, and male rats were subsequently housed socially with two to three animals from the same litter that belonged to the same experimental group under a $12 \mathrm{~h} / 12 \mathrm{~h}$ light/dark cycle (lights on at 7:00 $\mathrm{AM})$ at $23 \pm 1{ }^{\circ} \mathrm{C}$ and $55 \pm 5 \%$ humidity with food and water available ad libitum throughout the study. The experiments occurred between 9:00 AM and 5:00 PM. All of the experiments were performed in compliance with the recommendations of the Brazilian Society for Neuroscience and Behavior, which are based on the United States National Institutes of Health Guide for Care and Use of Laboratory Animals (publication no. 85-23, revised 1985) and were approved by the ethics committee of the Universidade Federal de São Paulo $(207 / 07)$. All efforts were made to minimize the number of animals used and their suffering.

\section{Intermittent hypoxia protocol}

Intermittent hypoxia was induced in a custom-built chamber $(30 \times 20 \times 20$ in; Oxycycler model A44X0, Biospherix, Redfield, NY, USA) connected to a supply of $\mathrm{O}_{2}$ and $\mathrm{N}_{2}$ gas. Sensors measured $\mathrm{O}_{2}$ concentration, $\mathrm{CO}_{2}$ concentration $(<0.01 \%)$, humidity $(40-50 \%)$, and temperature $\left(22-24^{\circ} \mathrm{C}\right)$. The inflow of $\mathrm{O}_{2}$ and $\mathrm{N}_{2}$ into the chamber was controlled by a computer programmed to produce cycles of $2 \mathrm{~min}$ of room air and $2 \mathrm{~min}$ of $10 \% \mathrm{O}_{2}$. The $\mathrm{IH}$ profile consisted of alternating room air and $10 \% \mathrm{O}_{2}$ every $2 \mathrm{~min}$, whereas treatment of the corresponding controls (i.e., the normoxic group) consisted of flushing room air into the chamber every 2 min (Gozal et al., 2001a; Perry, D’Almeida, Souza, Schoorlemmer, Columbari, \& Tufik, 2007).

On PD7, male rat pups were randomly assigned to three experimental groups as described by Decker et al. (2005): (1) intermittent hypoxia-inducing chamber, (2) normoxic chamber, and (3) control (unhandled). Once inside their respective chambers, the rat pups were exposed to either IH or normoxia during the subsequent $2 \mathrm{~h}$. The pups were then removed from the chambers and returned to their dams for $45 \mathrm{~min}$. The pups were then returned to their respective chambers and again exposed to $\mathrm{IH}$ or normoxia for another $2 \mathrm{~h}$. Upon completion of the second 2-h session, all of the pups were returned to their dams and left undisturbed until the following morning. During the next 4 days (PD8-11), the pups that were designated as hypoxic and normoxic were removed from their dams and placed in the hypoxia and normoxia chambers. During this period, hypoxic pups received $6 \mathrm{~h}$ of $\mathrm{IH}$ per day. They were exposed to $2 \mathrm{~h}$ of $\mathrm{IH}$ followed by a $45 \mathrm{~min}$ feeding and grooming session that was repeated three times each day. Normoxic pups were exposed to room air, and the non-handled controlpups were left undisturbed with their dams. The rats were subjected to this schedule during the light period (8:00 AM-5:00 PM). At the conclusion of the protocol on PD11, all of the pups were returned to their dams where they remained undisturbed until weaning on PD21. The rats were subsequently housed socially with two to three animals from the same litter that belonged to the same experimental group.They were tested for locomotor activity on PD56.

\section{Locomotoractivity protocol}

The openfield was a circular enclosure (arena) made of Plexiglas ( $60 \mathrm{~cm}$ diameter, $50 \mathrm{~cm}$ height) with a white acrylic floor divided into 12 equal sections $(13.3 \times$ $15.0 \mathrm{~cm}$ rectangles; Insight Equipment, Ribeirão Preto, SP, Brazil).

On PD56, the rats were tested in the open field to assess locomotor activity. The animals were individually placed in the center of the arena and allowed a $10 \mathrm{~min}$ period of free exploration, during which the number of crossings (i.e., the number of floor sections crossed with all four paws) was recorded. The behavior of the animals was recorded by a video camera (Safety View) positioned $60 \mathrm{~cm}$ above the arena, with the signal relayed to a video monitor in another room via a closed circuit. Testing was conducted under dim light (16 lux) to avoid the possible aversive effect of bright light. After each trial, the apparatus was cleaned with a $10 \%$ ethanol solution.

\section{Histological analyses: Nissl staining}

Upon completion of the locomotor activity test, the rats were deeply anaesthetized with urethane and intracardially perfused with $100 \mathrm{ml}$ phosphate-buffered saline (PBS) and $400 \mathrm{ml}$ of 4\% paraformaldehyde. Coronal brain sections (35 $\mu \mathrm{m}$ thick) were made with a cryostat between 0.98 and $-3.28 \mathrm{~mm}$ relative to bregma according to the stereotaxic coordinates of a rat brain atlas (Paxinos \& Watson, 2007). The sections were selected (12 per animal) by targeting the areas and subareas of the hippocampus (hilus, granular layer of the dentate gyrus, and CA1 and CA3 areas), nucleus accumbens shell and core, dorsal and ventral striatum, and prefrontal cortex (M2 and $\mathrm{Cg} 1)$. The selected sections were Nissl-stained with Cresyl violet $(0.2 \%$ Cresyl violet acetate in acetate buffer, $\mathrm{pH}$ 4.0), dehydrated with alcohol and xylene, and cover slipped.

The slides were examined using a light microscope (Nikon 80i), and images were captured and digitized using a Nikon ACT-1 v.2 system. Cell damage in the three selected subregions was estimated by counting the absolute number of surviving intact cells in the Nisslstained sections by two observers who were blind to 
the experimental conditions of the animals. The cells were manually counted under a microscope at $40 \times$ magnification in 10 random, non-overlapping fields for all of the animals in the three groups (control, normoxia, and hypoxia). Cells with somas $<3 \mu \mathrm{m}$ were considered glial or necrotic cells and excluded. The value attributed to each animal represents the mean value for all of the images taken from that animal.

\section{Statistical analysis}

One-factor (treatment) analysis of variance (ANOVA) was performed for the number of crossings and cell counts using Prism 4.0 software(GraphPad, San Diego, CA, USA), followed by the Tukey post hoc test. The alpha level was set at 0.05 for all of the statistical analyses.

\section{Results}

\section{Behavioral results}

One-way ANOVA revealed a significant effect of treatment on the number of crossings in the open field test $\left(F_{2,28}=9.16, p<0.05\right.$; Figure 1$)$. The posthoc analysis showed that both the hypoxia and normoxia groups exhibited an increase in the number of crossings compared with controls.

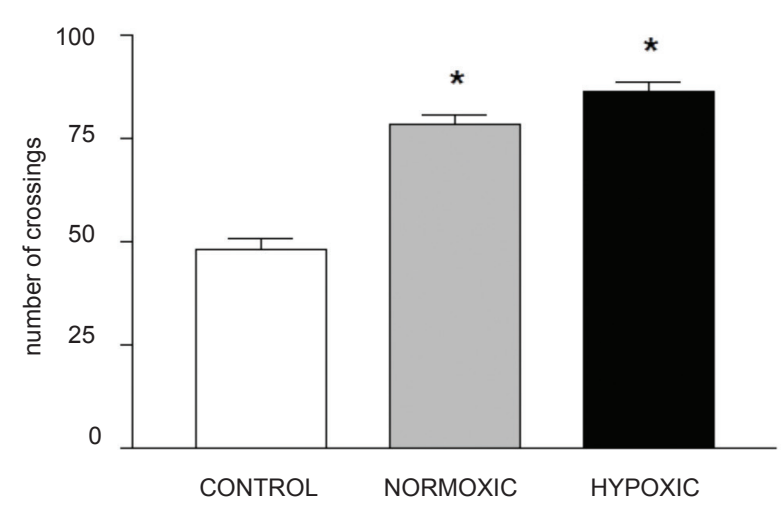

Figure 1. Mean + SEM number of crossings in the open field test in male Wistar rats in the control group $(n=9)$, normoxic group $(n=11)$, and hypoxic group $(n=11)$. ${ }^{*} p<0.05$ (oneway ANOVA followed by Tukey test).

\section{Neuronal cell damage}

Histological analyses were performed in Nisslstained sections to estimate neuronal death caused by IH by counting the number of intact cells in the studied brain regions. One-way ANOVA revealed a significant decrease in the number of intact cells in the hilus $\left(F_{2,31}=\right.$ 5.4, $p<0.05$; Figures 2A and 3A) and dorsal striatum $\left(F_{2,50}=3.6, p<0.05\right.$; Figures $2 \mathrm{~B}$ and $\left.3 \mathrm{~B}\right)$ in the hypoxia group compared with the control and normoxia groups, indicating cell loss. No significant cell loss was observed in the CA1 area $\left(F_{2,31}=0.37, p>0.05\right), \mathrm{CA} 3$ area $\left(F_{2,31}=\right.$ $0.20, p>0.05)$, prefrontal cortex $\left(F_{2,90}=2.3, p>0.05\right)$, or nucleus accumbens $\left(F_{2,33}=0.04, p>0.05\right)$ in these hypoxic rats.
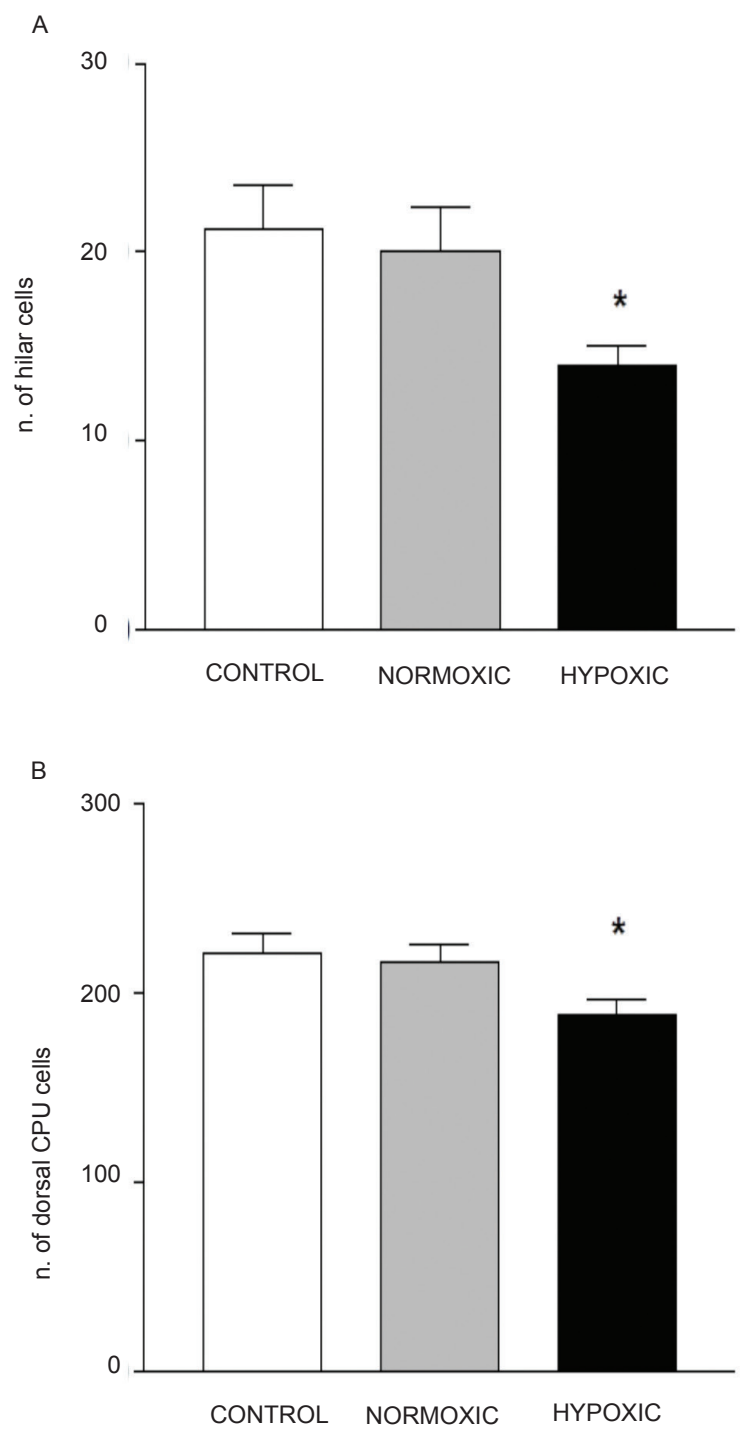

Figure 2. Cell survival estimated by quantifying the number of intact Nissl-stained cells. A significant reduction of the number of Nissl-stained was observed in the hilus of the hippocampal (A) and dorsal striatum (B) in the hypoxic group compared with the control group $(n=9)$; normoxic, $(n=11)$; hypoxic, $(n=11) ; \mathrm{p}<0.05$; (one-way ANOVA followed by Tukey test).

\section{Discussion}

Schizophrenia results from a cumulative interaction between numerous risk factors, some of which are neurodevelopmental. According to the neurodevelopmental hypothesis of schizophrenia, brain damage that occurs early in life increases the vulnerability of young adults to adverse events, thereby increasing the probability of clinical symptoms in adulthood (Jacob \& Beckmann, 1986, 1994; McNeil, Cantor-Graae, \& Ismail, 2000). An interaction between early neurodevelopmental disturbances and periadolescent brain maturation appears to be necessary to trigger the onset of full-blown psychotic behavior, which typically emerges during adolescence or early adulthood (Decker et al., 2003; Sandager-Nielsen et al., 2004; Meyer \& Feldon, 2010). 
A
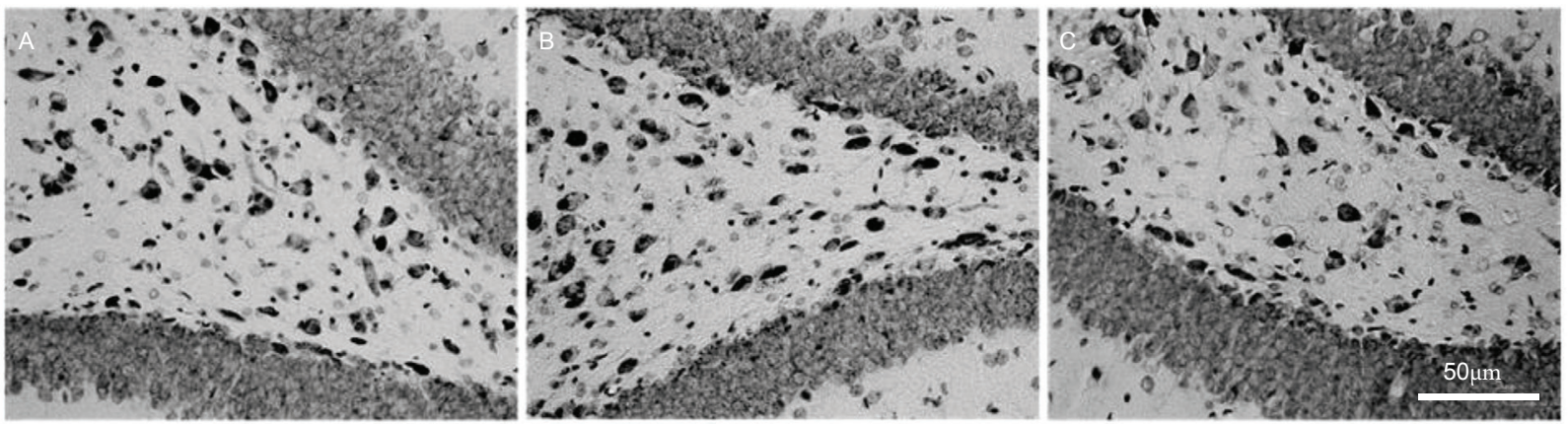

B
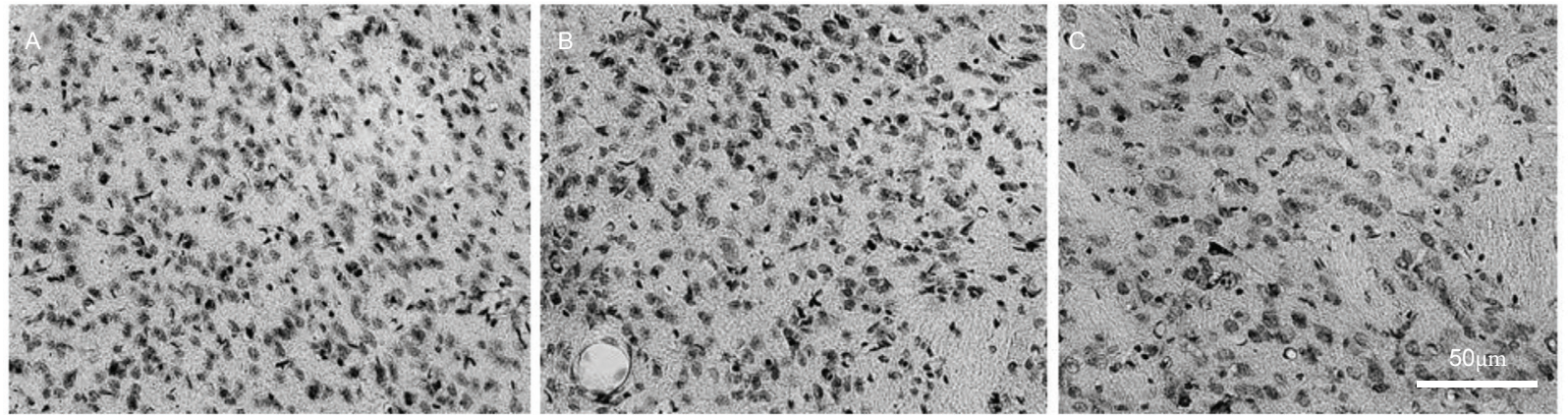

Figure 3. Photomicrograph of standard Nissl staining that shows the hilus of the hippocampus (A) and dorsal striatum (B) in the three groups: (A) control, (B) normoxic, and (C) hypoxic. Notice the reduction of the number of cells in the hilus and dorsal striatum in the hypoxic group. Magnification $=40 \times(A)$ and $20 \times(B)$. Scale bar $=50 \mu \mathrm{m}$.

The behavioral data obtained in the present study indicate that $\mathrm{IH}$ in rat pups during early developmental phases (PD7-11) led to an increase in locomotor activity when these animals were tested in early adulthood (PD56) compared with controls (Figure 1). Hyperactivity is thought to model some of the positive symptoms of schizophrenia and correlate well with psychomotor agitation observed in schizophrenia patients (Kilts, 2001). Our results are consistent with Decker et al. (2003, 2005), who observed an increase in the basal levels of locomotor activity in 42-dayold rats exposed to IH between PD7 and PD11. Other studies that used different protocols of hypoxia (Row et al., 2002) or anoxia (Dell'Anna, Calzolari, Molinari, Luvone, \& Calimici, 1991) also reported an increase in locomotor activity in adult animals exposed to these procedures early in life.

Interestingly, we observed an increase in the number of crossings in the open field in normoxic rats during adulthood compared with controls (Figure 1). One possible explanation for development hyperactivity in this group is the effect of stress associated with early maternal deprivation. Several animal studies have addressed the outcome of maternal deprivation and found long-term changes in brain neurochemistry and behavioral tasks (i.e., the open field, elevated plus maze, and forced swim test), suggesting increased anxiety, hyperactivity, and behavioral despair (George, Bordner, Elwafi, \& Siemen, 2010; Anand \& Scalzo, 2000; Hofer, 1980).

The hippocampus is a brain structure that has been traditionally associated with the neuropathology of schizophrenia. We found that post-hypoxic rats showed a reduction of the number of neurons restricted to the hilar region of the hippocampus (Figures $2 \mathrm{~A}$ and $3 \mathrm{~A}$ ). However, the changes in the number of hilar cells reported in this study were quite subtle and therefore difficult to compare directly with the effects of gross disruption of hippocampal circuitry. Some studies suggested that beyond the age of the animals tested in the present study, strain differences and the severity of hypoxia can be critical factors in determining the pattern of brain injury and extent to which neurological function will be affected, thus influencing the behavioral parameters evaluated in the present study. Moreover, the intensity and site of hippocampal damage might be related to the age at which the animals were tested (Simonová, Sterbová, Brozek, Komarék, \& Syková, 2003; Leonardo, Eakin, Ajmo, \& Gottschall, 2008). In the present study, the animals were exposed to a moderate level of hypoxia $\left(10 \% \mathrm{O}_{2}\right)$ on PD7-11 and evaluated on PD56. Perhaps a more intense hypoxic insult (e.g., 5\% $\mathrm{O}_{2}$; Polotsky et al., 2006) could lead to more severe neuronal damage compared with the present 
protocol. Indeed, Gozalet al. (2001a,b) showed that hypoxia induced neuronal death selectively in the CA1 region and cortex without modifying other hippocampal regions. Other studies also suggested that hypoxia induces selective neuronal death in the hippocampus (Feng, Zhao, Gurkoff, Van, Shahlaie, \& Lyeth, 2012), especially in the hilus (Schwartz, Massarweh, Vinters, \& Wasterlain, 1992).

Our findings demonstrated that hypoxia induced neuronal damage in the dorsal striatum (Figures $2 \mathrm{~B}$ and $3 \mathrm{~B}$ ). The striatum is one of the main regions that is damaged after neonatal hypoxia in rats (Rice, Vannucci, \& Brierley, 1981; Galvin \& Oorschot, 2003) and humans (Volpe, 2001). These data are consistent with other studies that reported neuronal loss in the striatum at different ages (Leonardo et al., 2008, Galvin $\&$ Oorschot, 2003). During the critical period of brain development, hypoxia can damage the functional integrity of the dopaminergic system, thus influencing relevant behavioral and neurochemical alterations (Decker et al., 2003, 2005). Row et al. (2002) verified that newborn rats (PD10) that were subjected to hypoxia exhibited an increase in locomotor activity when they reached 30 days of age.

Striatal dopaminergic dysfunction is commonly observed in schizophrenia and could be related to the dysfunction of dopamine-glutamate interactions. Usun, Eybrard, Meyer, \& Louilot (2013) showed that ketamine $(20 \mathrm{mg} / \mathrm{kg}$, i.c.) increased the release of dopamine in the dorsal striatum and increased locomotor activity in freely moving adult rats that had their prefrontal cortex inactivated by a microinjection of tetrodotoxin on PD8. The rats were monitored using in vivo voltammetry in the dorsal striatum. These data suggest that transient inactivation of the prefrontal cortex during early development results in greater behavioral and striatal dopaminergic reactivity to ketamine in adulthood.

Our results also showed that the nucleus accumbens core and shell and prefrontal cortex were not damaged after IH on PD7-11. Neurons in the hippocampus and dorsal striatum are less resistant to injury and die before neurons in the nucleus accumbens and prefrontal cortex during the critical developmental period evaluated in the present study (Golan \& Huleihel, 2006). Moreover, the intensity and duration of the hypoxic insult might influence the severity of damage (Ten, BradleyMoore, Gingrich, Stark, \& Pinsky, 2003). Moreover, other significant aspects, such as the unmyelinated state of the immature brain with a greater volume of the extracellular space, immaturity of the blood-brain barrier, cerebral blood flow during the first weeks of life, different glucose utilization, and immaturity of enzymes and receptors, are important for the cerebral response to injury and may affect the evolution of the injury (for review, see Hagberg, Bona, Gilland, \& Puka-Sundvall, 1997).

In conclusion, IH insult and maternal deprivation in rat pups on PD7-11 produced hyperactivity on PD56, which correlated well with psychomotor agitation observed in patients with schizophrenia. Post-hypoxic rats also exhibited a reduction of the number of neurons in the hilar region of the hippocampus and dorsal striatum, structures that have been traditionally associated with the neuropathology of schizophrenia. The nucleus accumbens and prefrontal cortex were not affected by IH. Several factors, such as the concentration of $\mathrm{O}_{2}$, duration of exposure to hypoxia, and age of the animals at the time of testing, can influence the response to hypoxia.

\section{Acknowledgments}

This work was sponsored by FAPESP (Proc. 2006/61223-3), CEPID (98/14303-3 to S. Tufik), and Associação Fundo de Incentivo à Pesquisa. K. Yamada, S. Satake, K. Garcia, and B.M. Longo were supported by FAPESP (Proc. 2009/07278-9, 2009/073470,2009/03616-7, and 2010/00120-8). J.C. Perry (Proc. 558924/2008-5), V. D'Almeida, and S. Tufik are recipients of CNPq fellowships.

\section{Declaration of interest}

The authors report no conflicts of interest. The authors alone are responsible for the content and writing of this paper.

\section{References}

Anand, K.J., \& Scalzo, F.M. (2000). Can adverse neonatal experiences alter brain development and subsequent behavior? Biology of the Neonate, 77(2),69-82.

Cannon, T.D. (1997). On the nature and mechanisms of obstetric influences in schizophrenia: a review and synthesis of epidemiologic studies. International Review of Psychiatry, 9(4), 387-397.

Cantor-Graae, E., Ismail, B., \& McNeil, T.F. (2000). Are neurological abnormalities in schizophrenic patients and their siblings the result of perinatal trauma? Acta Psychiatrica Scandinavica, 101(2), 142-147.

Decker, M.J., Hue, G.E., Caudle, W.M., Miller, G.W., Keating, G.L., \& Rye, D.B. (2003). Episodic neonatal hypoxia evokes executive dysfunction and regionally specific alterations in markers of dopamine signaling. Neuroscience, 117, 417-425.

Decker, M.J., Jones, K.A., Solomon, I.G., Keating, G.L., \& Rye, D.B. (2005). Reduced extracelullar dopamine and increased responsiveness to novelty: neurochemical and behavioural sequelae of intermittent hypoxia. Sleep, 28, 169-176.

Dell'Anna, M.E., Calzolari, S., Molinari, M., Luvone, L., \& Calimici, R. (1991). Neonatal anoxia induces transitory hyperactivity, permanent spatial memory deficits and CA1 cell density reduction in developing rats. Behavioural Brain Research, 45(2),125-134.

Feng, J.F., Zhao, X., Gurkoff, G.G., Van, K.C., Shahlaie, K., \& Lyeth, B.G. (2012). Post-traumatic hypoxia exacerbates neuronal cell death in the hippocampus. Journal of Neurotrauma, 29(6), 1167-1179.

Galvin, K.A., \& Oorschot, D.E. (2003). Continuous low-dose treatment with brain-derived neurotrophic factor or neurotrophin-3 protects striatal medium spiny neurons from mild neonatal hypoxia/ ischemia: a stereological study. Neuroscience, 118(4),1023-1032.

George, E.D., Bordner, K.A., Elwafi, H.M., \& Siemen, A.A. (2010). Maternal separation with early weaning: a novel mouse model of early life neglect. BMC Neuroscience, 11,123.

Golan, H., \& Huleihel, M. (2006). The effect of prenatal hypoxia on brain development: short- and long-term consequences demonstrated in rodent models.Developmental Science, 9, 338-349.

Gozal, D., Daniel, J.M., \& Dohanich, G.P. (2001a). Behavioral and anatomical correlates of chronic episodic hypoxia during sleep in the rat. Journal of Neuroscience, 21, 2442-2450.

Gozal, E., Row, B.W.,Schurr, A., \& Gozal, D. (2001b). Developmental differences in cortical and hippocampal vulnerability to intermittent hypoxia in the rat. Neuroscience Letters, 305, 197-201. 
Hagberg, H., Bona, E., Gilland, E., \& Puka-Sundvall, M. (1997). Hypoxia-ischaemia model in the 7-day-old rat: possibilities and shortcomings. Acta Paediatrica Supplement, 422, 85-88.

Hofer, M.A. (1980). Effects of reserpine and amphetamine on the development of hyperactivity in maternally deprived rat pups. Psychosomatic Medicine, 42(5), 513-520.

Jakob, J. \& Beckmann, H. (1986). Prenatal developmental disturbances in the limbic allocortex in schizophrenics. Journal of Neural Transmission, 65, 303-326.

Jakob, J. \& Beckmann, H. (1994). Circumscribed malformation and nerve cell alterations in the entorhinal cortex of schizophrenics: pathogenetic and clinical aspects. Journal of Neural Transmission: General Section, 98, 83-106.

Juarez, I., Silva-Gomez, A.B., Peralta, F., \& Flores, G. (2003). Anoxia at birth induced hyperresponsiveness to amphetamine and stress in postpubertal rats. Brain Research, 992(2), 281-287.

Kilts, C.D. (2001). The changing roles and targets for animal models of schizophrenia. Biological Psychiatry, 50, 845-855.

Leonardo, C.C., Eakin, A.K., Ajmo, J.M., \& Gottschall, P.E. (2008). Versican and brevican are expressed with distinct pathology in neonatal hypoxic-ischemic injury. Journal of Neuroscience Research, 86(5), 1106-1114.

McNeil, T.F. (1995). Perinatal risk factors and schizophrenia: selective review and methodological concerns. Epidemiologic Reviews, 17(1), 107-112.

McNeil, T.F., Cantor-Graae, E., \& Ismail B. (2000). Obstetric complications and congenital malformation in schizophrenia. Brain Research Reviews, 31, 166-178.

Meyer, U. \& Feldon, J. (2010). Epidemiology-driven neurodevelopmental animal models of schizophrenia. Progress in Neurobiology, 90, 285-326.

Paxinos, G. \& Watson, C. (2007). The rat brain in stereotaxic coordinates, $6^{\text {th }}$ edition. San Diego: Academic Press.

Perry, J.C., D'Almeida, V., Souza, F.G., Schoorlemmer, G.H., Colombari, E., \& Tufik, S. (2007). Consequences of subchronic and chronic exposure to intermittent hypoxia and sleep deprivation on cardiovascular risk factors in rats. Respiratory Physiology and Neurobiology, 156, 250-258.
Polotsky, V.Y., Rubin, A.E., Balbir, A., Dean, T., Smith, P.L., Scwartz, A.R., \& O'Donnell, C.P. (2006). Intermittent hypoxia causes REM sleep deficits and decreases EEG delta power in NREM sleep in the C57BL/6J mouse. Sleep Medicine, 7(1), 7-16.

Rice, J.E., Vannucci, R.C., \& Brierley, J.B. (1981). The influence of immaturity on hypoxic-ischemic brain damage in the rat. Annals of Neurology, 9, 131-141.

Row, B.W., Kheirandish, L., Neville, J.J., \& Gozal, D. (2002). Impaired spatial learning and hyperactivity in developing rats exposed to intermittent hypoxia. Pediatric Research, 52, 449-453.

Sandager-Nielsen, K., Andersen, M.B., Sager, T.N., Werge, T., \& Scheel-Kruger, J. (2004). Effects of postnatal anoxia on striatal dopamine metabolism and prepulse inhibition in rats. Pharmacology Biochemistry and Behavior, 77(4), 767-774.

Schwabe, K., Klein, S., \& Koch, M. (2006). Behavioural effects of neonatal lesions of the medial prefrontal cortex and subchronic pubertal treatment with phencyclidine of adult rats. Behavioural Brain Research, 168(1), 150-160.

Schwartz, P.H., Massarweh, W.F., Vinters, H.V., \& Wasterlain, C.G. (1992). A rat model of severe neonatal hypoxic-ischemic brain injury. Stroke, 23(4), 539-546.

Simonová, Z., Sterbová, K., Brozek, G., Komarék, V., \& Syková, E. (2003). Postnatal hypobaric hypoxia in rats impairs water maze learning and morphology of neurones and macroglia in cortex and hippocampus. Behavioural Brain Research, 141(2), 195-205.

Ten, V.S., Bradley-Moore, M., Gingrich, J.A., Stark, R.I., \& Pinsky, D.J. (2003). Brain injury and neurofunctional deficit in neonatal mice with hypoxic-ischemic encephalopathy. Behavioural Brain Research, 145, 209-219.

Usun, Y., Eybrard, S., Meyer, F., \& Louilot, A. (2013). Ketamine increases striatal dopamine release and hyperlocomotion in adult rats after postnatal functional blockade of the prefrontal cortex. Behavioural Brain Research, 16, 229-237.

Vaillancourt, C., \& Boksa, P. (2000). Birth insult alters dopaminemediated behavior in a precocial species, the guinea pig: implications for schizophrenia. Neuropsychopharmacology, 23(6), 654-666

Volpe, J.J. (2001). Neurology of the newborn, 4th edition. Philadelphia: W.B. Saunders. 